\title{
An evidence-based policy brief: improving the quality of postnatal care in mothers 48 hours after childbirth
}

\section{Betty Sakala, Ellen Chirwa}

University of Malawi Kamuzu College of Nursing

\begin{abstract}
Introduction
Abstract

Malawi is experiencing slow progress in postnatal care of mothers within the first 48 hours after

childbirth. Malawi Demographic and Health Survey (MDHS) 2015-16 reported a slow progress in postnatal care of mothers in the first 48 hours at $42 \%$ from $41 \%$ in 2010 despite a high number of institutional births. This is a critical period as a large proportion of maternal deaths occur during this period, currently at 439 per 100,000 live births. During postnatal care the mother is given important information to assist in caring for herself and her baby. The lack of well documented guidelines and funding to employ more midwives to manage mothers in postnatal ward contributes to poor quality of postnatal care.

Methods

This is an evidence-based policy brief that was prepared to inform policy makers, health workers, clients, community and other stakeholders to consider the available evidence about the impact of the suggested options in order to improve postnatal care.

Results

Several factors that contribute to low utilization of postnatal care among mothers after childbirth were identified. Factors included lack of clear guidelines on postnatal care, shortage of skilled health workers and inadequate resources.

Conclusion

Implementation of the identified policy options may improve postnatal care.
\end{abstract}

Key words: Policy brief, postnatal care, evidence-based, policy options, guidelines, skilled health workers

\section{Introduction}

-Low coverage of postnatal care

There is little progress in postnatal checks within 2 days after childbirth in health facilities: 42\% in 2016 from 41\% in 2010 .

There is slow progress in the postnatal care of mothers within the first 48 hours after childbirth. Malawi Demographic and Health Survey (MDHS) 2015-16 reported slow progress in the postnatal care of mothers in the first 48 hours at $42 \%$ from $41 \%$ in 2010 with Mangochi district registering the lowest percentage of mothers $(16.8 \%)$ attending postnatal care within 48 hours despite a high number of institutional births, $90.6 \%$. This is a critical period as a large proportion of maternal deaths occur during this period currently at 439 per 100,000 live births ${ }^{1}$. Postnatal care is important to identify and treat complications after childbirth. In addition, the mother is given important information to assist in caring for herself and her baby. The lack of well documented guidelines and funding to employ more midwives to manage mothers in postnatal wards contributes to the poor quality of postnatal care.

\section{Policy Options}

\section{Monitoring of postnatal care service before discharging mothers after childbirth}

The proposed options can greatly contribute to the quality of postnatal care and the last option will help to facilitate postnatal care of mothers who fail to deliver at a health facility.
1. Availability of postnatal care guidelines at all health facilities providing maternal and neonatal care services: the information to include all areas to be assessed during physical examination of a mother and baby before discharge. 2. Allocating full time midwives in the postnatal wards: this option targets health facilities that do not allocate midwives to care for mothers who have undergone normal childbirth but their main focus is mothers who developed complication. 3. Community awareness on importance of postnatal care and referral of mothers who deliver before reaching the health facility.

4. Introducing postnatal outreach clinics or home visits to target mothers who deliver in other places apart from a health facility and those discharged within 24-48 hours after childbirth.

\section{Implementation considerations}

Several factors should be taken into account to promote postanatal care services:

- Provision of clear guidelines

- Sensitazation of communitie on the importance of postanatal care.

Emphasis on policies and programmes that advocate for good quality of postnatal care will greatly contribute to the reduction of maternal mortality and morbidity.

- Several factors should be taken into account in order to promote postnatal care services: provision of clear postnatal guidelines; sensitisation of communities on the importance of postnatal care; priority of care to be extended to mothers who have undergone normal childbirth. 
- Factors that may hinder utilization of postnatal services may include: negative attitudes of midwives as regards to mothers who give birth on their way to hospital or at home; inadequate material and human resource to offer quality care, limited financial resource to hire trained personnel and upgrade infrastructure. Suggestions to overcome the identified barriers are included in the pages to follow.

\section{The Problem}

\section{Low coverage of postnatal care of mothers within the first 48 hours after childbirth in Malawi}

Most of the complications leading to postpartum maternal mortality and morbidity arise during labour and delivery, and during the first one to two weeks following delivery. The postnatal period is a very critical phase in the lives of a mother and her baby as major physiological and psychological changes occur during this period. Yet, this is the most neglected time for the provision of quality services ${ }^{2}$. In addition, the lack of appropriate care given to mothers and newborns during this period may result to mortality and morbidity. Furthermore, postnatal care is given reduced attention as compared to antenatal care and childbirth. For instance, in the National Sexual and Reproductive Health Rights (SRHR) ${ }^{3}$ the objectives placed emphasis on Focused Antenatal Care and other services. In addition, the maternal and neonatal policy statements and strategies stress on antenatal, neonatal and post abortion care but postnatal care is not well stipulated.

The 2015-16 MDHS reported a steady decline of maternal mortality from 675 per 100, 000 live births in 2010 to 439 per 100,000 in 2016 with haemorrhage being the major cause of maternal deaths. Despite this development, Malawi is still reporting the highest maternal mortality ratio in sub-Saharan Africa ${ }^{4}$.

\section{Size of the problem}

There is slow progress in the provision of postnatal care services to mothers in the first 48 hours of child birth $41 \%$ in 2010 to $42 \%$ in $2016^{1}$. $60 \%$ of maternal deaths occur within 24 hours after childbirth ${ }^{5}$. It is reported that almost $50 \%$ of mothers who delivered in the hospital did not receive any postnatal care from 48 hours, one week and six weeks. The burden of low utilization of postnatal care may increase due to increase in institutional births currently at $91 \%{ }^{1}$ against a vacancy rate of about $63 \%$ nurse/midwives ${ }^{4}$.

\section{Factors underlying the problem}

There are several factors that contribute to low utilization of postnatal care services among mothers after childbirth:

\section{Organizational factors contributing to low utilization of postnatal care}

There are no clear guidelines on postnatal care as each hospital discharges mothers according to availability of space. There is need to standardise the discharge planning process for all hospitals for better client outcomes. Most health facilities do not allocate skilled health providers in postnatal wards and mostly the same providers in labour ward or postnatal ward who handle complicated births also look after mothers who had uncomplicated births. As such, unskilled health providers are assigned to assist in care of mothers with uncomplicated birth leading to poor quality of care.

\section{Client and skilled healthcare providers}

There are inadequate refresher courses and a lack of mentorship in relation to postnatal care as much emphasis is placed on labour and birth complications. Few skilled providers are allocated to manage postnatal mothers and their babies. As such, there is heavy workload leading to provider fatigue that may result in provision of low quality care to mothers and their babies.

\section{Financing arrangements}

Priority in funding may not be allocated to mothers who have undergone normal childbirth, as such, early identification of complications among these mothers may be a problem.

\section{Four policy options}

The Malawi Ministry of Health recommends that postnatal care should be offered to all mothers who deliver at a health facility within the first 24 hours after childbirth while those who gave birth at other places other than a health facility should be referred within 12 hours to have postnatal care by a skilled health provider. ${ }^{1}$ WHO recommends that health facilities should offer additional postnatal care for all mothers and newborns on day 3 (48-72 hours) and between days 7-14 after birth, and six weeks after childbirth ${ }^{2}$. The four policy options may be adopted independently or could complement one another. Quality of care may be improved to reduce adverse birth outcomes through adequate staffing level and provision of relevant postnatal care policies.

\section{Policy option 1: Availability of a comprehensive postnatal care tool at all health facilities offering maternal and neonatal care services}

This policy option will include all areas of the body to be assessed during physical examination of a mother and her baby before discharge. These may include identification and management of complications for mother and her baby such as postpartum depression, haemorrhage and neonatal infections ${ }^{5,6}$. Evidence shows that $10-15 \%$ of postnatal mothers experience postpartum depression and symptoms are not identified early due to inadequate counselling and physical examination by health workers ${ }^{6}$.

Despite having postnatal care guidelines in Malawi Reproductive Health Service Delivery Guidelines ${ }^{7}$, it is not well known in the dissemination of the guidelines. There is little progress on postnatal checks within 48 hours after childbirth form $41 \%$ in 2010 to $42 \%$ in $2016^{1}$ yet $90 \%$ of deliveries are conducted by skilled birth attendants.

The mother and her family require more information on care of the baby and herself within the first week after childbirth to identify any adverse birth outcomes ${ }^{5}$. These include control of mother's bleeding, absence of signs of infections on mother and baby and ability of the baby to breastfeed well.

\section{Policy option 2: Allocating full time midwives in the postnatal wards to manage postnatal mothers and their babies}

This option targets health facilities that do not allocate midwives to care for mothers who had uncomplicated childbirth. Most health facilities allocate one midwife to care for mothers in labour ward as well as postnatal ward. As a result, care is compromised because these midwives are tired and overworked ${ }^{8}$. $\mathrm{WHO}^{2}$ recommends that standard of care for mothers and newborns should be provided in health 
facilities according to WHO guidelines, that is full clinical examination after childbirth and before discharge.

There is need to advocate for adequate deployment and retention of midwives in addition to attractive salaries as well as good living conditions for those in rural areas so as to have midwives who will be responsible for providing standard care to all mothers and babies irrespective of their birth outcomes.

\section{Policy option 3: Community awareness on importance of postnatal care and referral of mothers who deliver before reaching the bealth facility.}

Community awareness on postnatal care is very important as the community may get specific information that is crucial in early identification of danger signs that warrant referral to a health facility ${ }^{5}$. In addition, the communities may be assisted to form community support groups that will be responsible for sharing important messages pertaining to postnatal care. Collaborating with community members may enhance knowledge of health promotion activities pertaining to mothers and their babies. The communities will also be empowered to refer mothers who deliver before reaching the health facility for postnatal care including counselling on issues pertaining to postnatal care.

Lack of community awareness on importance of postnatal care may contribute to late identification of complications as communities feel that all mothers who had uncomplicated childbirth may be attended to by traditional birth attendants as postnatal care is only necessary for mothers who develop obstetric complications ${ }^{9,11}$. More interventions are required to improve community awareness and perception on postnatal care services in order to improve care and these interventions should mainly target rural women ${ }^{10}$ thereby improving the utilisation of postnatal care.

Literature shows that lack of awareness on postnatal care services contributes to low utilisation of postnatal care services since it is attached to care of new born babies only because of vaccination schedules ${ }^{11}$. A study conducted in Malawi on community scorecard on reproductive healthrelated outcomes emphasised the need for community awareness to improve reproductive health-related outcomes $^{12}$. It is believed that when communities are aware of the available services, they are empowered and take full responsibility to reinforce the importance of reproductive health services such as postnatal care.

\section{Policy option 4: Introducing postnatal outreach clinics or home visits}

This policy option will target women who deliver in other places apart from a health facility and those discharged within 24-48 hours after childbirth. WHO ${ }^{2}$ recommends that home visits for postnatal mothers and their babies be conducted in the first week after childbirth. This is important for checking the general wellbeing as well as conducting general assessments to identify adverse postnatal outcomes that may warrant referral to a health facility such as haemorrhage, infections, anaemia and depression. In the 2017-2022 Health Sector Strategic Plan 11, maternal sepsis was identified as one of the priority areas requiring prompt interventions. Therefore, it is necessary to intensify postnatal home visits so that mothers are identified early for prompt management. Postnatal sepsis is also the second direct cause of maternal deaths at $18.9 \%{ }^{13}$. For this reason, it is highly recommended that the community health package should include postnatal care home visits as one of the priority areas.

Many studies have shown the importance of postnatal home visits. It is indicated that mothers prefer to report to traditional birth attendants for postnatal care services since they are readily available unlike skilled birth attendants'. In a related study conducted in Sweden, it was also reported that mothers preferred to have home-based postnatal care offered by midwives unlike other health workers ${ }^{14}$ and this may be applicable in Malawi if there are clear developed strategies on home-based maternal and neonatal care. In addition, postnatal home visit was considered to be crucial in early identification of mothers who are prone to postpartum depression such as first time mothers ${ }^{15}$. The authors argued that mothers' self confidence in caring for themselves and their babies may be uplifted through home visits. Several authors also emphasised the importance of postnatal home visits $^{12}$. Gullo et al reported that home visits for postnatal care improved women satisfaction in reproductive health services by $6 \%$. In related studies by Dodge et $\mathrm{al}^{16}$ and Olayinka et $\mathrm{al}^{17}$ on impact of postnatal care home visit on obstetric care and awareness and barriers to utilization of maternal health care services, it was reported that home visiting helps to identify mothers with obstetric complications who may need referral for further management. The authors recommended more allocation of resources to support maternal and infant home visits. Evidence has shown that postnatal home visits may also benefit the baby. According to research findings, health education on breastfeeding offered during postnatal home visit improved women's knowledge on breastfeeding. As such there was improved infant health outcome in the first year of life ${ }^{18,19,16}$.

Due to shortage of skilled health workers in Malawi, it may not be possible to have midwives making home visits. But with the high number of mothers who did not receive postnatal check (50\%) despite delivering in a health facility; it is important to conduct postnatal home visit to capture these mothers. We therefore recommended that community nurse midwife technicians or community midwifery assistants be deployed in the communities to offer such services and this may require proper planning and adequate resource allocation.

\section{Implementation considerations}

Improving postnatal care can be achieved through reinforcement and development of the already existing interventions, guidelines and tools as outlined by WHO; employing more midwives; empowering mothers, families and communities on Maternal and Neonatal Health issues; and introduction of postnatal outreach clinics or postnatal home visits.

Key considerations for creation of an enabling environment for the provision of postnatal care services include:

- Well established postnatal care services at district hospitals and health centres

- Reviewing national guidelines and policies on management of postnatal mothers and babies

- Health promotion programs to increase awareness on importance of postnatal care services

- Reviewing policies to improve better working environment for midwives

- Conduct more local research studies on postnatal care services 


\section{Barriers to improving postnatal care}

Several studies have reported on the impact of the above barriers on postnatal care. For instance, the shortage of midwives needs special consideration to improve the quality of postnatal care. A report by White Ribbon Alliance ${ }^{20}$ indicated that Malawi is not meeting the recommended ratio of midwife to women which is at 1:5. Current statistics indicate a ratio of 1:10 according to number of midwives registered with Nurses and Midwives Council of Malawi. This figure may not give a true reflection because it is counting all midwives who have paid their registration fee. It is well known that all registered midwives are not practicing midwifery in the clinical area because some are working in other departments or Non-Governmental Organisations (NGO). Despite increasing intake of students in training colleges, there is still poor retention of midwives due to internal and external migration of midwives.

White Ribbon Alliance indicted that poor remuneration and working conditions are the main contributing factors to internal and external migration of these midwives. For this reason, White Ribbon Alliance recommended that government should increase national budget for training and deployment of more midwives in maternity departments, adequate remuneration for midwives and adequate material and non-material resources. Bradley et $\mathrm{al}^{8}$ also concurred with White Ribbon Alliance observations on critical shortage of midwives. It is reported that in other places, two midwives were seen working during day shift and one midwife on night shift covering both labour and postnatal wards. In another scenario, one midwife was reported to cover labour ward, postnatal, nursery, antenatal ward and theatre during night shift. In a related development, the State of the Worlds' Midwifery report indicated that many midwives are deployed in urban areas yet a large number of the population is in rural areas. This implied that rural women were denied chances of care provided by professional midwives ${ }^{21}$.

Since findings indicate that shortage of midwives and lack of resources still exists in the hospitals, the possibility of advocating for increasing the length of stay to minimum of 48 hours is a challenge. These findings are similar with those found in Uttar Pradesh ${ }^{22}$ on challenges of early postnatal discharge. The study findings indicated that women had postnatal discharges as early as six hours after childbirth. This was done because of lack of beds and other resources at the hospital. It was also discovered that even women who stayed longer in the hospital were not given quality care because of work overload and inadequate resources. Varma et $\mathrm{al}^{22}$ therefore recommended postnatal home visits in order to identify danger signs and possibility of early referral in case of complications.

Furthermore, a study conducted in Australia on quality and safety of postnatal discharge ${ }^{23}$ indicated that lack of proper clinical guidelines in postnatal discharge planning process contributed to serious risks to the mother and infant. It was discovered that although midwives were scheduled to conduct home visits during postnatal, several challenges were encountered such as high workload due to shortage of midwives and lack of transport. As a result of these developments, the authors recommended a minimum stay of 48 hours for mothers leaving in remote areas. A study conducted on impact of length of postpartum hospital stay ${ }^{24}$ revealed that prolonged hospital stay improved the health of the mother and infant. Among the health benefits, there was improved breast feeding initiation, early identification of complications such as haemorrhage, postpartum depression and reduction of readmissions. As such, the authors recommended a minimum postnatal stay of 48 hours to minimise complications of childbirths.Evidence regarding key barriers to improving postnatal care for mothers and babies and strategies to address the identified barriers are summarised in Table 1

\section{Table 1: Barriers to improving postnatal care and proposed} strategies to overcome them

\begin{tabular}{|l|l|}
\hline Barrier & Implementation strategies \\
\hline $\begin{array}{l}\text { Inadequate knowledge } \\
\text { on importance of post- } \\
\text { natal care services by } \\
\text { communities }\end{array}$ & $\begin{array}{l}\text { Awareness campaigns in } \\
\text { communities to sensitize them on } \\
\text { importance of postnatal care } \\
\text { Encourage ownership of } \\
\text { reproductive health services } \\
\text { particularly those related to } \\
\text { postnatal care }\end{array}$ \\
\hline $\begin{array}{l}\text { Inadequate knowledge } \\
\text { of health care providers } \\
\text { of WHO guidelines on } \\
\text { postnatal care }\end{array}$ & $\begin{array}{l}\text { Conduct in-service trainings and } \\
\text { mentorship specifically for midwives } \\
\text { on proposed WHO guidelines on } \\
\text { management of postnatal mothers } \\
\text { and babies } \\
\text { Regular supportive supervisions } \\
\text { by advanced midwives to boost } \\
\text { confidence of junior midwives } \\
\text { Review existing guidelines and } \\
\text { policies on postnatal care }\end{array}$ \\
\hline Shortage of midwives & $\begin{array}{l}\text { Adequate remuneration, material } \\
\text { and non-material resources are } \\
\text { essential to motivate midwives } \\
\text { Provide good working environment } \\
\text { both at district and health centre } \\
\text { level } \\
\text { Support increased funding to } \\
\text { employ more midwives and for } \\
\text { improved postnatal care activities }\end{array}$ \\
\hline
\end{tabular}

\section{Proposed strategies to improve postnatal care}

After going through the options recommended from different studies, we suggest trying policy option 4 that advocates introduction of postnatal outreach clinics or home visits specifically for postnatal care. The authors of this policy brief would like to recommend Ministry of Health to consider utilizing the already existing Malawi Community Based Maternal New born Care (CBMNC) package introduced by Save the Children ${ }^{25}$. The authors of CBMNC evaluated three districts (Thyolo, Dowa and Chitipa) that were utilizing the CBMNC package using Health Surveillance Assistants (HSAs). The findings indicated that $44 \%$ of mothers who informed the HSAs of childbirth received the required postnatal home visits within the first week of childbirth as compared to $5 \%$ of those who did not contact the HSAs.

Considering the workload of HSAs, we suggest use of community nurse midwives and community midwifery assistants to conduct postnatal outreach clinics to identify complications early and make timely referral. The HSAs will be allowed to focus on their intended tasks such as water and sanitation activities, under five clinic, immunizations and community meetings. The midwives will be working in coordination with the HSAs because they will be working in the same catchment area. The study results also indicated that the CBMNC package had the potential to improve maternal 
and neonatal health. The authors therefore suggested increasing number of HSAs and improving communication from health facilities on current births in order to increase coverage of postnatal care. It is for this reason that the authors of this policy brief feel that deploying the already existing community midwife assistants and community midwife technicians by $\mathrm{MoH}$ would improve postnatal care. The $\mathrm{MoH}$ could lobby for funds from donors who fund HSAs to use the suggested cadres in order to relieve HSAs of work overload and concentrate on their intended tasks.

\section{Next Steps}

The aim of this policy brief is to inform deliberations about the existing postnatal care guidelines and policies with reference to available evidence relating to the problem and possible solutions. The suggested options may guide further actions to improve postnatal care services. Thus necessitating:

- Further research on each of the policy options to attain high quality postnatal care.

- Reviewing of postnatal guidelines and policy to align with WHO recommendations.

- Strengthening feasibility of postnatal care home visits with reference to CBMNC package.

\section{Acknowledgement}

The primary author is a $\mathrm{PhD}$ in midwifery student at Kamuzu College of Nursing and the policy brief was prepared as a fulfilment for a course in Advanced Leadership, Management and Policy for Health Professionals. The author would like to thank Professor Ellen Chirwa of Kamuzu College of Nursing for her contributions on the policy brief.

\section{References}

1. National Statistical Office (NSO) [Malawi], and ICF. Malawi Demographic and Health survey, 2015-16.Zomba, Malawi, and Rockville, Maryland, USA. NSO and ICF, 2017. www.DHS.com

2. World Health Organization. WHO recommendations on postnatal care of the mother and newborn. World Health Organization, 2014. www.who.int

3. Government of Malawi, Sexual and Reproductive Health Rights 2017-22

4. Government of Malawi, Health Sector Strategic Plan II 2017-22

5. World Health Organization. WHO technical consultation on postpartum and postnatal care, 2010. www.who.int

6. Singh A, Yadav A, Singh A. Utilization of postnatal care for newborns and its association with neonatal mortality in India: an analytical appraisal. BMC Preg CB. 2012; 12(1):33 https://doi.org/10.1186/14712393-12-33

7. Government of Malawi. Reproductive Health Guidelines 2014-19. 2014

8. Bradley S, Kamwendo F, Chipeta E, Chimwaza W, de Pinho H, McAuliffe E. Too few staff, too many patients: a qualitative study of the impact on obstetric care providers and on quality of care in Malawi. BMC Preg CB. 2015; 15(1): 65 DOI 10.1186/s12884-015-0492-5

9. Titaley CR, Hunter CL, Heywood P, Dibley, MJ. Why don't some women attend antenatal and postnatal care services? A qualitative study of community members' perspectives in Garut, Sukabumi and Ciamis districts of West Java Province, Indonesia. BMC Preg CB.2010; 10(1): $61 \mathrm{https}: / /$ doi.org/10.1186/1471-2393-10-61
10. Worku AG, Yalew AW, Afework MF. Factors affecting utilization of skilled maternal care in Northwest Ethiopia: a multilevel analysis. BMC Int health Hum Rig.2013; 13(1): 20 https://doi.org/10.1186/1472698X-13-20

11. Tesfahun F, Worku W, Mazengiya F, Kifle M. Knowledge, perception and utilization of postnatal care of mothers in Gondar Zuria District, Ethiopia: a cross-sectional study. MCH J. 2014; 18(10): 2341$51 \mathrm{https} / / /$ doi.org/10.1007/s10995-014-1474-3

12. Gullo S, Galavotti C, Kuhlmann AS, Msiska T, Hastings P, Marti CN. Effects of a social accountability approach CARE's Community Score Card on reproductive health-related outcomes in Malawi: A cluster-randomized controlled evaluation. PLoS one.2017; 12(2): e0171316 https://doi.org/10.1371/journal.pone.0171316

13. Government of Malawi. Emergency Obstetric and Neonatal Care (EmONC) Report; 2015

14. Johansson K, Aarts C, Darj E. First-time parents' experiences of home-based postnatal care in Sweden. Up J Med Sc.2010; 115(2):131$37 \mathrm{https} / / /$ doi.org/10.3109/03009730903431809

15. Leahy-Warren P, McCarthy G, Corcoran P. First-time mothers: social support, maternal parental self-efficacy and postnatal depression. J Clin Nurs. 2012; 21(3-4):388-97 doi: 10.1111/j.1365-2702.2011.03701.x

16. Dodge KA, Goodman WB, Murphy RA, O’Donnell K, Sato J. Randomized controlled trial of universal postnatal nurse home visiting: impact on emergency care. Ped. 2013; 132(Supplement 2): S140-46 https://dx.doi.org/10.1542\%2Fpeds.2013-1021M

17. Olayinka OA, Achi OT, Amos AO, Chiedu, EM. Awareness and barriers to utilization of maternal health care services among reproductive women in Amassoma community, Bayelsa State. Int $\mathbf{J}$ Nurs Mid.2014; 6(1): 10-15 DOI:10.5897/IJNM2013.0108

18.Gogia S, Sachdev HS. Home visits by community health workers to prevent neonatal deaths in developing countries: a systematic review. Bul WHO. 2010; 88(9): 658-66 doi:10.2471/BLT.09.069369

19. Aksu H, Küçük M, Düzgün, G. The effect of postnatal breastfeeding education/support offered at home 3 days after delivery on breastfeeding duration and knowledge: a randomized trial. J Mat-Fet Neo Med. 2011; 24(2):354-61 https://doi.org/10.3109/14767058.2010.497569

20. White Ribbon Alliance. Investing in midwifery in Malawi: Delivering on Commitments-Policy Brief. 2014

21. Nove A. Midwifery in Malawi: In-depth country analysis. Document prepared for the State of the World's Midwifery Report. 2011 Unpublished. Retrieved from http://www. helse-bergen. no/en/OmOss/ Avdelinger/internasjonalt-samarbeid/prosjekt/malawi/Documents/ Malawi $\%$ 20the $\%$ 20state $\%$ 20of $\%$ 20the $\%$ 20worlds $\%$ 20midwifery. pdf.

22. Varma DS, Khan M E, \& Hazra, A. Increasing postnatal care of mothers and newborns including follow-up cord care and thermal care in rural Uttar Pradesh. J Fam Wel. 2010; 56:31-42 https://www. researchgate.net/profile/Deepthi_Varma/publication/269986292

23. Bar-Zeev SJ, Barclay L, Farrington C, Kildea S. From hospital to home: the quality and safety of a postnatal discharge system used for remote dwelling Aboriginal mothers and infants in the top end of Australia. Mid.2012; 28(3): 366-73 https://doi.org/10.1016/j. midw.2011.04.010

24. Almond D, Doyle JJ. After midnight: A regression discontinuity design in length of postpartum hospital stays. Amer Eco J: Eco Pol. 2011; 3(3):1-34 https://www.nber.org/papers/w13877.pdf

25. Greco G, Daviaud E, Owen $\mathrm{H}$ et al. Malawi three district evaluation: Community-based maternal and newborn care economic analysis. Health Pol Plan. 2017 